\title{
ANÁLISE COMPARATIVA DOS PRODUTOS MODIS ECOLOGIA PARA O MONITORAMENTO BIOFÍSICO AMBIENTAL DO BIOMA CERRADO
}

\author{
Manuel Eduardo Ferreira ${ }^{1}$, Laerte Guimarães Ferreira ${ }^{1}$, Alfredo R. Huete $^{2}$ e Alejandro Alvarado Peccinini ${ }^{3}$ \\ Recebido em 30 janeiro, 2006 / Aceito em 21 junho, 2006 \\ Received on January 30, 2006 / Accepted on June 21, 2006
}

\begin{abstract}
The Brazilian Cerrado is an extensive and complex biome, characterized by rapid and abrupt land cover changes. Due to its dimensions and physiognomic variations, the Cerrado plays an important role regarding the water, energy, and carbon fluxes at both the regional and global scales. Therefore, the correct understanding of the structure and ecological functioning of this biome, particularly in the temporal domain, is of great importance. With this respect, in this study we compared the seasonal response and land cover discrimination of the major MODIS (MODerate resolution Imaging Spectroradiometer) "biophysical" indices: the normalized difference vegetation index (NDVI), the enhanced vegetation index (EVI), the leaf area index (LAl), and the fraction of absorbed photosynthetically active radiation (fAPAR). In spite of the fact the four indices showed similar temporal trends, the LAl showed the highest sensitivity to the seasonal variations of the natural and converted landscapes. On the other hand, the NDVI showed the best performance regarding land cover discrimination. Our results suggest a synergistic approach concerning the MODIS biophysical / ecological variables for land cover assessments and environmental monitoring of the Cerrado biome.
\end{abstract}

Keywords: Cerrado biophysical parameters, MODIS Ecology, MOD13, MOD15.

RESUMO. 0 Cerrado brasileiro é um extenso e complexo bioma, caracterizado por mudanças rápidas e bruscas na cobertura vegetal. Devido a suas dimensões e variações fitofisionômicas, o Cerrado desempenha um papel importante no que diz respeito aos fluxos de água, energia e carbono, tanto em escala regional quanto global. Deste modo, a correta compreensão do funcionamento estrutural e ecológico deste bioma, particularmente no domínio temporal, é de grande importância. Sobre isso, neste trabalho, comparou-se a resposta sazonal e potencial de discriminação de uso das terras dos principais índices "biofísicos" do sensor MODIS (MODerate resolution Imaging Spectroradiometer): 0 índice de vegetação por diferença normalizada (NDVI), o índice de vegetação realçado (EVI), o índice de área foliar (LAI) e a fração absorvida de radiação fotossinteticamente ativa (fAPAR). Os quatro índices exibiram comportamentos temporais similares, contudo, o LAl mostrou sensibilidade mais elevada às variações sazonais de paisagens naturais e antrópicas. Por outro lado, o NDVI apresentou a melhor performance referente à discriminação de uso das terras. Os resultados sugerem uma abordagem sinérgica em relação às variáveis biofísicas / ecológicas do MODIS para a avaliação da cobertura vegetal, do uso das terras e para o monitoramento ambiental do bioma Cerrado.

Palavras-chave: Parâmetros biofísicos do Cerrado, MODIS Ecologia, MOD13, MOD15.

\footnotetext{
${ }_{1}$ Universidade Federal de Goiás (UFG), Instituto de Estudos Sócio Ambientais (IESA), Laboratório de Processamento de Imagens e Geoprocessamento (LAPIG), Campus II, Cx. Postal 131, 74001-970 Goiânia, G0, Brasil. Tel: (62) 3521-1096; Fax: (62) 3521-1077 - E-mails: manuel@iesa.ufg.br, laerte@iesa.ufg.br ${ }^{2}$ University of Arizona (UofA), Terrestrial Biophysics and Remote Sensing Lab, SWES Dept., 429 Shantz Bldg., \#38, Tucson, AZ 85721-0038, USA. E-mail: ahuete@ag.arizona.edu

${ }^{3}$ Agência Goiana do Meio Ambiente (AGMA), 11a. Avenida, 1272, Setor Universitário, 74605-060 Goiânia, G0, Brasil. Tel: (62) 265-1386

-E-mail: alejandro@agenciaambiental.go.gov.br
} 


\section{INTRODUÇÃO}

0 ambiente savânico no Brasil, conhecido localmente como Cerrado, é um bioma extenso e complexo, podendo ser descrito como um mosaico de tipos vegetacionais, verticalmente estruturado por espécies herbáceas, arbustivas e arborescentes (Figura 1). Este gradiente natural, determinado, entre outros fatores, pela disponibilidade de água e fertilidade do solo, é marcado por uma conspícua sazonalidade entre as estações chuvosa (outubro a março) e seca (abril a setembro) (Dias, 1992; Pivello \& Coutinho, 1996).

Este bioma, presente em $25 \%$ do território nacional, desempenha um papel importante nos balanços de energia, água e carbono da região, atuando como um sumidouro de gases de efeito estufa, tal como o CO, $\mathrm{CO}_{2}$ e $\mathrm{CH}_{4}$ (Monteiro, 1995). Sua elevada biodiversidade, atualmente estimada em seis mil espécies vegetais vasculares (Mendonça et al., 1998), é favorecida pela presença de três das maiores bacias hidrográficas da América do Sul (Tocantins, São Francisco e Paraná) (Latrubesse et al., 2005), além de uma extensa diversidade de solos, geologia e queimadas naturais (Eiten, 1972).

Apesar da sua importância sócio-ambiental, o Cerrado encontra-se ainda pouco valorizado em termos de conservação, apresentando apenas $1,7 \%$ de sua área assegurada na forma de parques ou reservas (Brasil, 2003). Estima-se que atividades como a pecuária e a agricultura sejam responsáveis, até 0 momento, pela conversão de, aproximadamente, $40 \%$ de sua área original (Myers et al., 2000; Sano et al., 2002).

0 sensoriamento remoto, tido como uma fonte contínua e regular para a obtenção de dados da superfície terrestre, pode prover um monitoramento sistemático desse bioma, como o que vem sendo realizado pelo PRODES ${ }^{1}$ Digital (Duarte et al., 2003) e, mais recentemente, pelo SIAD ${ }^{2}$ (Sistema Integrado de Alerta de Desmatamentos) na região da Amazônia Legal (Ferreira et al., 2006).

Entre os sensores orbitais atualmente em uso pela comunidade científica, destaca-se o MODIS (MODerate resolution Imaging Spectroradiometer), concebido para fornecer informações sobre a biosfera terrestre com cobertura global a cada dois dias e resoluções espaciais variando de $250 \mathrm{~m}$ a $1000 \mathrm{~m}$ (Justice et al., 1998; Huete et al., 2002). Uma característica importante em relação ao MODIS é o fato dos dados serem disponibilizados prontos para o uso, i.e., georreferenciados e corrigidos para efeitos atmosféricos, na forma de diferentes produtos, entre os

\footnotetext{
${ }^{1}$ Projeto de Estimativa do Desflorestamento Bruto da Amazônia (versão digital).

${ }^{2}$ Mais informações em www.ufg.br/siad.
}

${ }^{3} \mathrm{NDVI}$ (Normalized Difference Vegetation Index), EVI (Enhanced Vegetation Index), LAI (Leaf Area Index) e fAPAR (fraction of Absorbed Photosynthetically Active Radiation). quais destacam-se aqueles para aplicações em ecologia terrestre (Tabela 1).

Produtos como o MOD13 (Índices de Vegetação NDVI e EVI) e 0 M0D15 (Índice de Área Foliar - LAl e Fração Absorvida de Radiação Fotossinteticamente Ativa - fAPAR) (Myneni et al., 2002) podem auxiliar no monitoramento efetivo e sistemático de todo o Cerrado, bem como possibilitar que a análise das paisagens natural e antrópica dos seus vários ecossistemas passe a ser realizada numa escala multitemporal, melhorando assim o entendimento deste bioma no que diz respeito à sua estrutura, funcionamento e função ecológica.

Especificamente, o presente trabalho analisa, de forma comparativa, a resposta dos produtos LAl/AAPAR (MOD15A2) e os produtos NDVI/EVI (MOD13A2) ${ }^{3}$, produzidos a cada 16 dias, ambos com um $1 \mathrm{~km}$ de resolução, à sazonalidade das várias paisagens típicas do bioma Cerrado no Estado de Goiás, incluindo as fitofisionomias nativas e convertidas (i.e. áreas de pastagens e agricultura). Da mesma forma, 0 desempenho destes índices e respectivas séries temporais quanto à discriminação destas várias paisagens é também avaliado.

\section{METODOLOGIA \\ Área de estudo}

A área de estudo engloba o Estado de Goiás, correspondendo aos tilesh13v10 e h12v10 do sensor MODIS. Foram escolhidos seis pontos de controle para extração das seguintes classes de amostras: 1) Floresta Estacional (Parque Ecológico Altamiro de Moura Pacheco); 2) Agricultura; 3) Pastagem; 4) Campo limpo/Campo sujo (Parque Nacional das Emas); 5) Campo Cerrado (Parque Nacional de Brasília) e 6) Cerrado denso (Parque Nacional Chapada dos Veadeiros) (Figura 2).

\section{Obtenção e análise dos dados}

Para cada produto analisado nesta pesquisa, isto é, NDVI e EVI (MOD13A2) e LAl e fAPAR (MOD15A2), foram adquiridos 24 mosaicos completos para 0 Estado de Goiás, a cada 16 dias, entre dezembro de 2002 e dezembro de 2003. Dessa forma, foi possível comparar qualitativa e quantitativamente 0 comportamento radiométrico de cada alvo, em função do respectivo parâmetro ecológico considerado e da variação da sazonalidade, ao longo de um ciclo hidrológico completo. Para cada classe de amostra (ex. pastagem) e ao longo de cada um dos 24 mosaicos de 


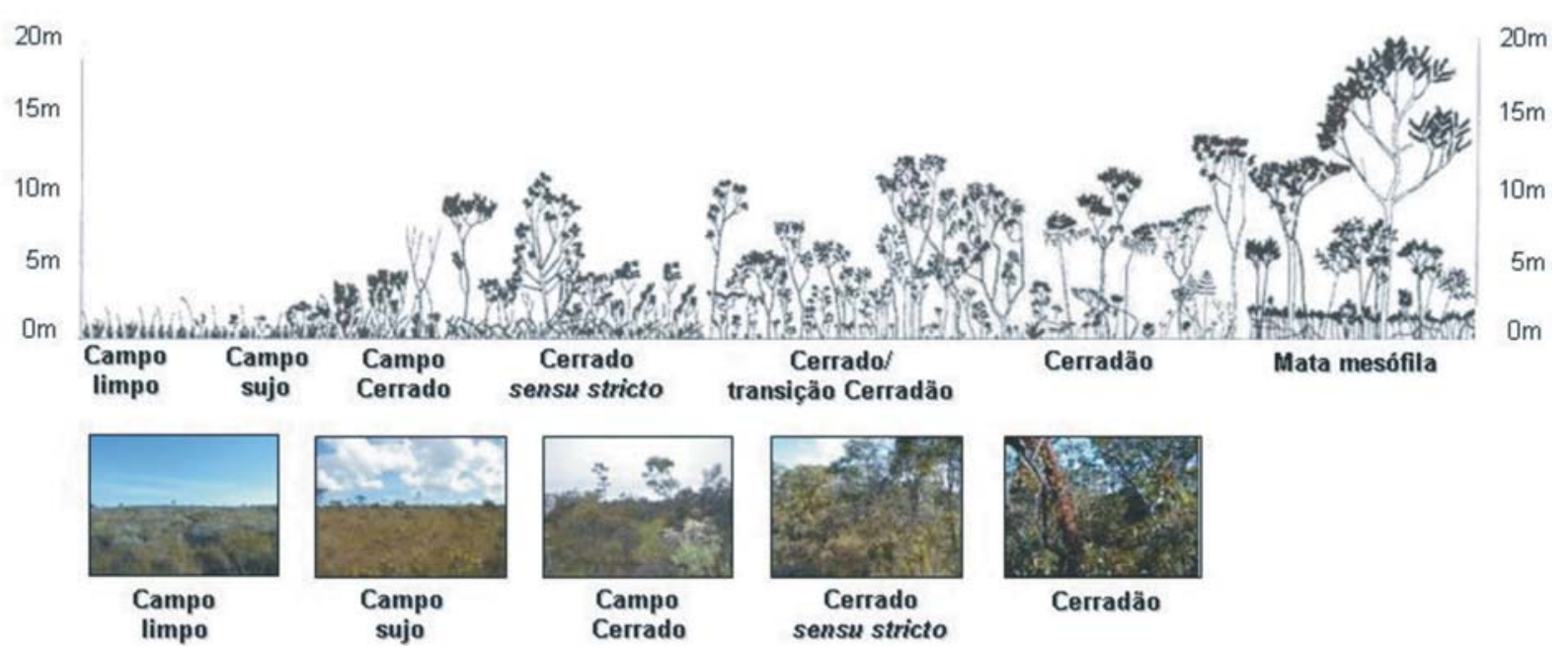

Figura 1 - Principais fitofisionomias encontradas no bioma Cerrado. Modificada de Bitencourt et al. (1997).

Tabela 1 - Produtos MODIS para aplicações terrestres (MODLAND), entre os quais destacam-se os índices de vegetação (MOD13) e 0 índice de área foliar / fração absorvida da radiação fotossinteticamente ativa (MOD15), analisados neste estudo.

\begin{tabular}{|c|c|c|}
\hline $\begin{array}{c}\text { Balanço de } \\
\text { energia e radiação } \\
\text { da superfície }\end{array}$ & $\begin{array}{l}\text { Produtos de } \\
\text { vegetação e } \\
\text { ecologia }\end{array}$ & $\begin{array}{c}\text { Cobertura e uso } \\
\text { da terra }\end{array}$ \\
\hline Refletância & Índices de Vegetação & $\begin{array}{c}\text { Cobertura/Mudanças } \\
\text { na Cobertura }\end{array}$ \\
\hline $\begin{array}{c}\text { Temperatura da } \\
\text { Superfície }\end{array}$ & $\begin{array}{l}\text { Índice de Área Foliar / } \\
\text { Fração de Radiação } \\
\text { Absorvida } \\
\text { Fotossinteticamente } \\
\text { Ativa }\end{array}$ & $\begin{array}{l}\text { Cobertura Vegetal / } \\
\text { Conversões }\end{array}$ \\
\hline Albedo & Produtividade Primária & $\begin{array}{c}\text { Anomalias Termais / } \\
\text { Fogo }\end{array}$ \\
\hline \multicolumn{3}{|c|}{ Aplicações Gerais } \\
\hline $\begin{array}{c}\text { Avaliação Física de } \\
\text { Processos Superficiais }\end{array}$ & $\begin{array}{c}\text { Caracterização e } \\
\text { Funcionamento de } \\
\text { Ecossistemas; Padrão } \\
\text { de Produtividade } \\
\text { Sazonais }\end{array}$ & $\begin{array}{c}\text { Estimativas de Carbono / } \\
\text { Desenvolvimento } \\
\text { Sustentável }\end{array}$ \\
\hline
\end{tabular}

imagens MODIS para Goiás, foi coletado um grupo de 10 pixe/s (Figura 2).

A resposta sazonal de cada fitofisionomia e classe de uso da terra, em relação a cada um dos parâmetros radiométricos considerados, foi analisada qualitativamente a partir de curvas temporais, definidas em função dos valores médios para cada grupo de pixels.
Por outro lado, análises de caráter mais quantitativo foram obtidas a partir das diferenças relativas entre 0 Dia do Ano (DA) 113 (período chuvoso) e 241 (período seco), bem como através do coeficiente de variação para as respostas médias dos nove mosaicos MODIS compreendidos entre os Dias do Ano 113 e 241 (i.e. período de maior variaçãa sazonal). Estas duas medidas normalizadas, de tal forma a facilitar a comparação de variáveis 

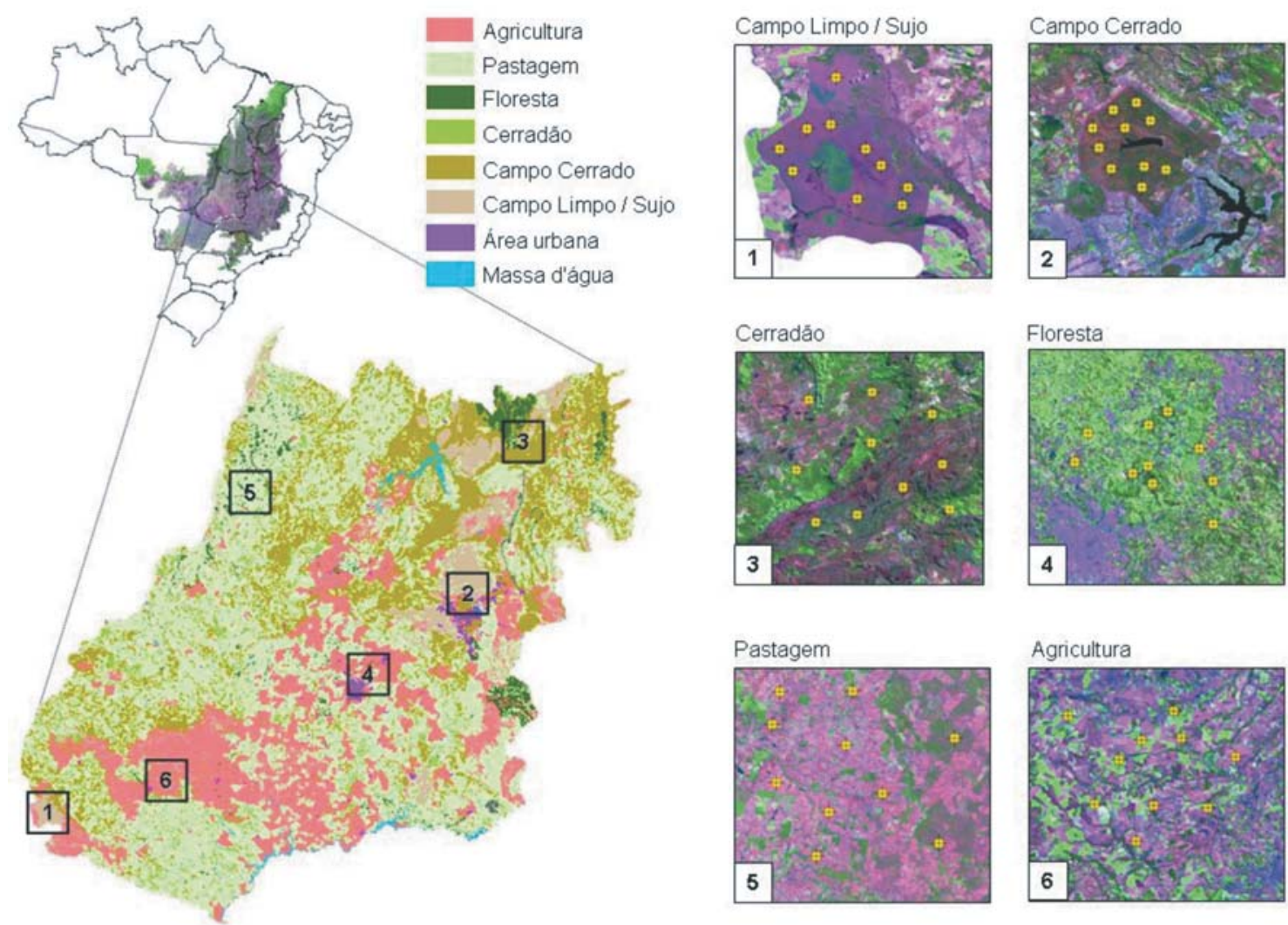

Figura 2 - Mapa de cobertura dos solos para o Estado de Goiás (Área de estudo) e locais de amostragem (identificados sobre imagens Landsat) para as seis paisagens naturais e antrópicas analisadas neste estudo.

distintas (i.e. NDVI, EVI, LAl e fAPAR), são descritas nas Eqs. (1) e (2):

$$
\begin{gathered}
\operatorname{VSR}(I V, L A I, f A P A R)= \\
{\left[\frac{(D A 113-D A 241)}{D A 113}\right] \times 100} \\
C V(I V, L A I, f A P A R)=\frac{\frac{\sqrt{\sum_{i=1}^{n}}\left(x_{i}-\bar{x}\right)^{2}}{(n-1)}}{\bar{x}}
\end{gathered}
$$

Onde: VSR: Variação Sazonal Relativa; $D A$ : Dia do Ano; $I V, L A I, f A P A R$ : índices analisados; $C V$ : Coeficiente de Variação.

A possibilidade de discriminação destas paisagens, a partir das respectivas séries temporais analisadas neste estudo, foi avaliada por meio de uma análise de discriminante baseada na distância de Mahalanobis (Schowengerdt, 1997).

\section{RESULTADOS E DISCUSSÃO}

\section{Resposta dos Índices MOD13 e MOD15 à Dinâmica Sazonal das Paisagens}

Em relação às classes de uso antrópico (agricultura e pastagem), um padrão similar de curvas foi observado entre os índices avaliados, fato este explicado pela ocorrência dominante de apenas uma espécie vegetal anual em cada grupo amostrado, ou seja, na área de agricultura uma formação de monocultura, e na área de pastagem a presença de gramínea.

As variações sazonais destas duas classes ocorrem, principalmente, devido ao ciclo de vida das espécies anuais, o qual se inicia na época chuvosa e termina na época seca e, no caso das pastagens, pelo efeito do sobrepastejo causado pelos rebanhos bovinos. A Figura 3 ilustra tais resultados.

Observando as classes de Campo limpo/Campo sujo, e Campo Cerrado (Figura 4), constata-se que, assim como nas classes de uso antrópico, estes ambientes apresentam uma queda significativa de biomassa durante a época seca, verificada para 


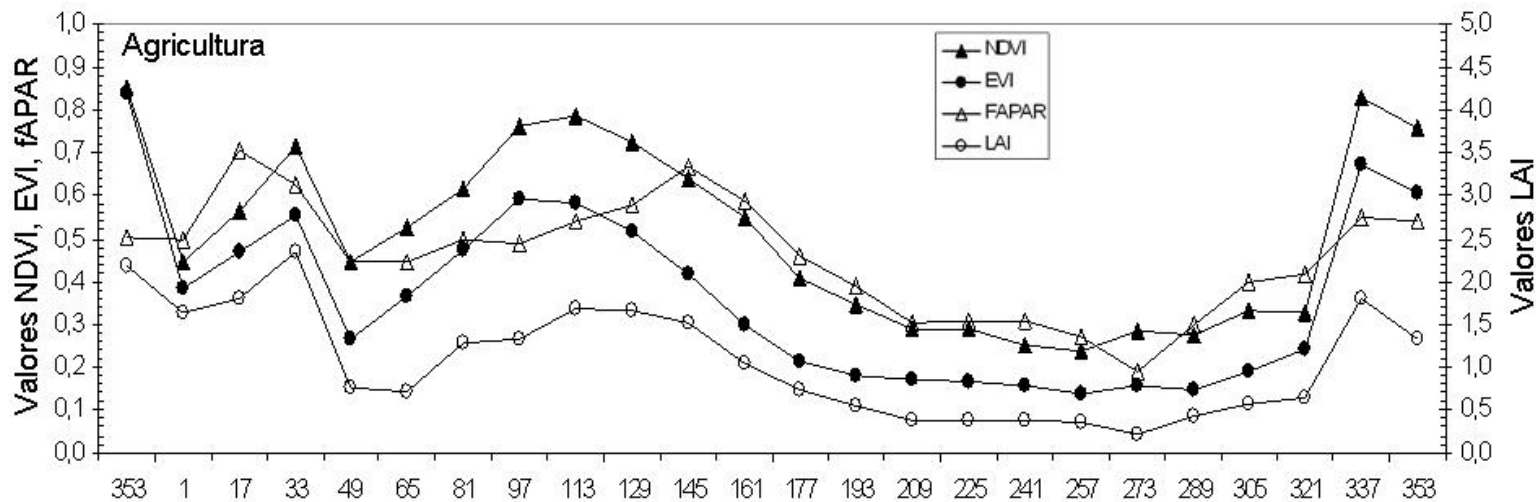

Dia do ano (dez. 2002 - dez. 2003)

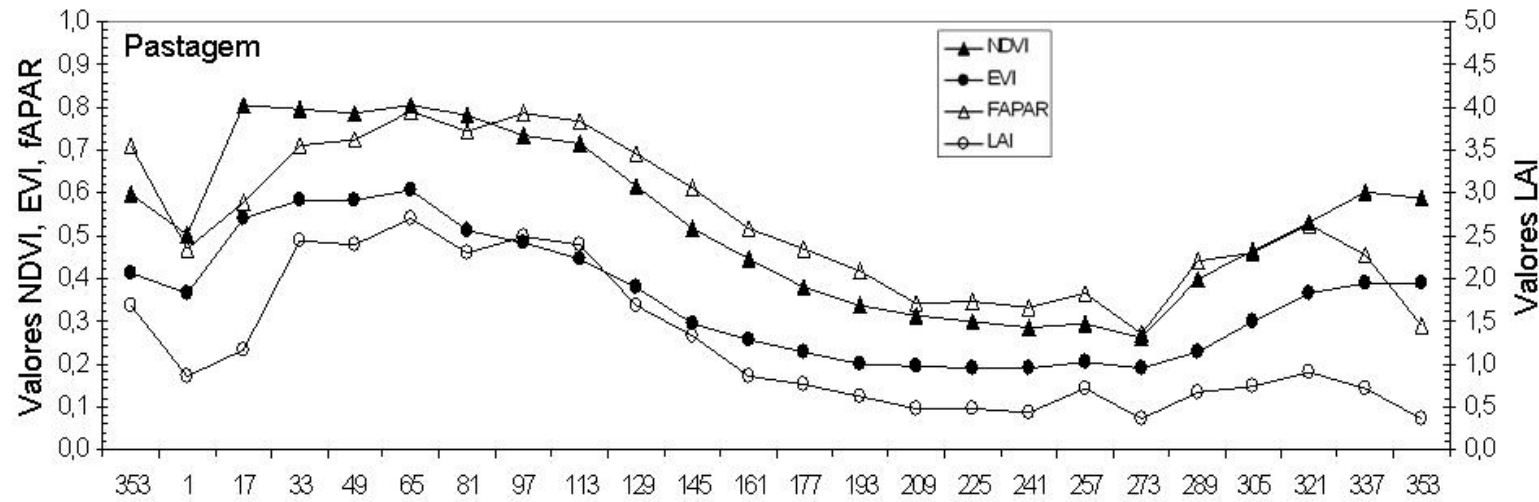

Dia do ano (dez. $2002-$ dez. 2003)

Figura 3 - Resposta sazonal dos índices NDVI, EVI, LAI e fAPAR para as classes de uso antrópico (agricultura e pastagem).

todos os quatro índices. Especificamente no caso dos Campos limpo e sujo, tal fato se deve à predominância de formações herbáceas anuais, que perdem 0 seu componente fotossintetizante durante a época seca. Em relação ao Campo Cerrado, comparativamente, observa-se em todos os índices uma queda menor de valores no período seco, provavelmente devido à ocorrência de maior biomassa estrutural (ex. ramos, troncos), presente durante todo 0 ano. Também é importante ressaltar a maior oscilação relativa dos valores correspondentes ao LAl, e em menor grau ao fAPAR, o que pode estar associado à uma maior sensibilidade destes índices quanto aos fenômenos de florescimento e rebrotamento.

Em fato, e ao contrário do observado para as classes agricultura e pastagem, ainda que todos os índices apresentem uma mesma tendência de variação (i.e. diminuição de valores da época chuvosa para a época seca), os padrões de resposta temporal dos produtos MOD13 e MOD15 para as classes Campo limpo e Campo Cerrado são ligeiramente distintos.
A análise das classes arbórea/florestal indica uma menor variação sazonal relativa devido à maior presença tanto de biomassa verde quanto estrutural ao longo do ano. A Figura 5 ilustra este fato, confirmado por todos os índices. Por outro lado, é importante ressaltar as nítidas diferenças, principalmente no caso da classe floresta, observadas entre as curvas dos produtos MOD13 e MOD15. Da mesma forma que observado para as classes de Cerrado herbáceo e arbustivo, os índices fAPAR, e em particular o LAl, apresentam maiores variações em suas respostas ao longo do ano.

Em concordância ao que vem sendo demonstrado em outros trabalhos de caráter comparativo (Cohen et al., 2003; Wang et al., 2004), observa-se de modo geral que, à medida que se desloca em direção à fitofisionomias mais arborescentes, há uma tendência crescente de diferenciação entre os padrões de resposta temporal dos índices de vegetação (EVI e NDVI), mais relacionados à parâmetros superficiais da vegetação (ex. \% de cobertura verde), e entre as curvas dos produtos LAI e fAPAR, mais 


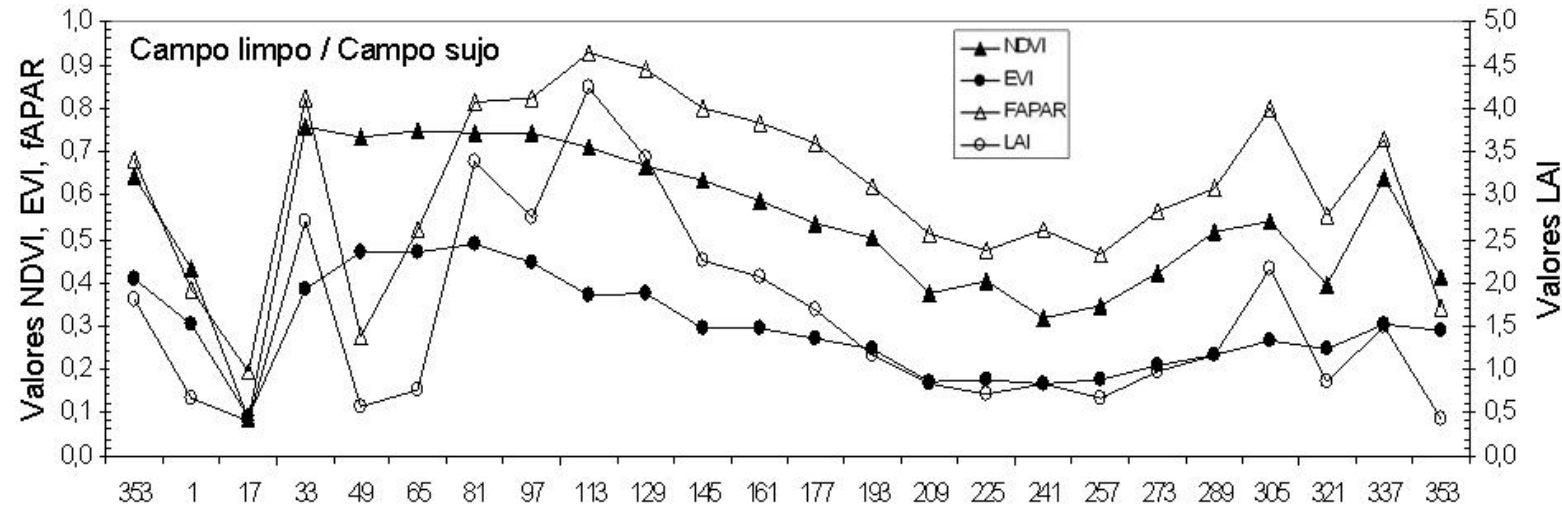

Dia do ano (dez. $2002-$ dez. 2003)

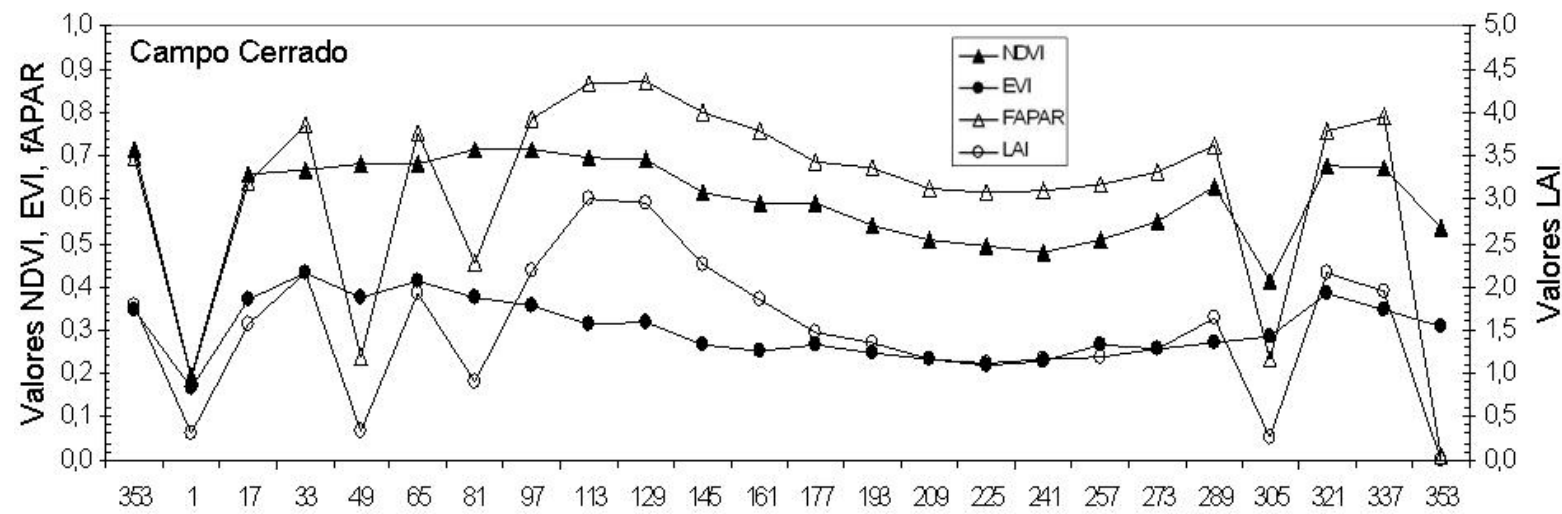

Dia do ano (dez. 2002 - dez. 2003)

Figura 4 - Resposta sazonal dos índices NDVI, EVI, LAl e fAPAR para classes Campo limpo/Campo sujo e Campo Cerrado (variações abruptas nas respostas radiométricas entre os dias 1 e 65 - i.e. janeiro e fevereiro - se devem a presença de nuvens residuais nas respectivas imagens).

relacionados a aspectos estruturais e funcionais da vegetação.

As menores variações sazonais observadas para as fitofisionomias mais arborescentes e os maiores valores de variação verificados para as classes de uso antrópico são também observadas através das medidas de variação radiométrica relativa entre os dias do ano 113 (período chuvoso) e 241 (período seco) (Figura 6). Assim, enquanto as classes agricultura, pastagem e campo limpo/campo sujo apresentam variações radiométricas superiores a 50\%, as três classes arborescentes apresentam diferenças radiométricas em média inferiores a 30\%. Na Figura 6, também é possível constatar uma maior sensibilidade do índice LAl, comparativamente ao fAPAR, à dinâmica temporal das paisagens, ao contrário dos resultados apresentados por Fensholt et al. (2004), cujo estudo em área de savana demonstrou a baixa eficiência do LAI na caracterização sazonal. Da mesma forma, entre os índices de vegetação, também se observa um desempenho em geral ligeiramente melhor do EVI comparativamente ao NDVI,
0 que é coerente aos resultados encontrados por Ferreira et al. (2003) para dados MODIS simulados a partir de levantamentos aero-espectrorradiométricos.

A maior sensibilidade do LAl sobre os demais índices, e do EVI em relação ao NDVI, às variações sazonais das paisagens é também corroborada através das imagens mostradas na Figura 7 , bem como pelos respectivos coeficientes de variação (CVs), os quais, ao retratarem as diferenças de cada índice em relação aos vários períodos do ano analisados (i.e. do dia 113 ao dia 241), constituem-se em uma importante medida do desempenho destes índices em responderem às condições das várias fitofisionomias (Figura 8). Conforme observado na Figura 8, maiores valores de CV, entre 0,6 e 0,7 são observados para as fitofisionomias com menores quantidades de biomassa estrutural. É interessante observar que estes valores são muito parecidos com os valores apresentados na Figura 6. De fato, os dois gráficos (i.e. Figuras 6 e 8) apresentam padrões de variação e valores relativos muito 

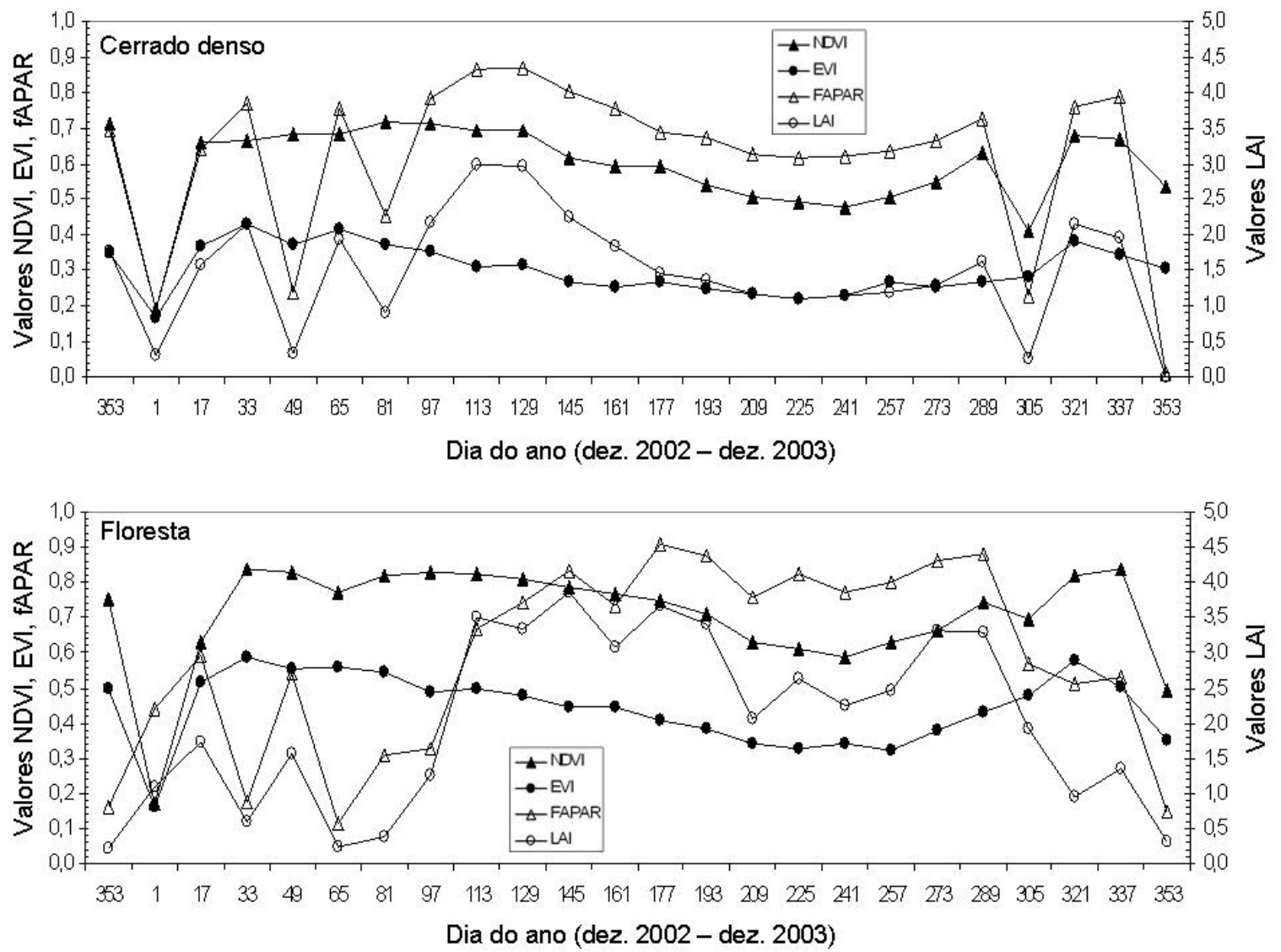

Figura 5 - Resposta sazonal dos índices NDVI, EVI, LAI e fAPAR para as classes Cerrado denso e Floresta (variações abruptas nas respostas radiométricas entre os dias 1 e 65 - i.e. janeiro e fevereiro - se devem a presença de nuvens residuais nas respectivas imagens).

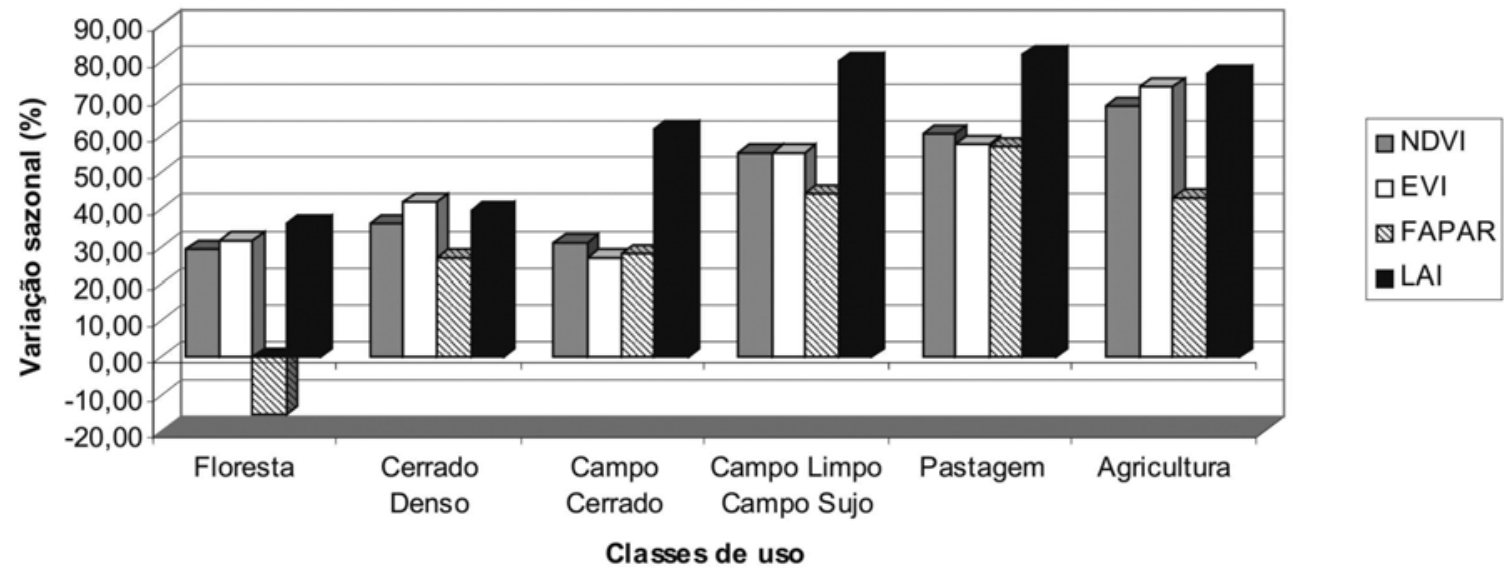

Figura 6 - Variação sazonal de valores dos produtos MODIS para cada classe amostrada. 
semelhantes. Por outro lado, e considerando o fato das medidas de variações relativas e CVs serem obtidas a partir de metodologias e conjuntos de dados distintos, as semelhanças observadas, ao contrário de redundantes, reforçam os resultados e conclusões apresentadas.

\section{Discriminação das Paisagens através de séries tempo- rais MOD13 e MOD15}

Em relação à possibilidade de discriminação das principais paisagens encontradas no bioma Cerrado (i.e. paisagens arborescentes, arbustivas/herbáceas e convertidas para pastagens cultivadas e agricultura), os quatro índices MODIS avaliados neste estudo apresentaram desempenho bastante semelhante (Tabela 2), com índice geral de amostras corretamente classificadas superior a 80\%. Ainda assim, é interessante ressaltar o melhor desempenho do NDVI (97\%) e o baixo desempenho relativo do LAI (83\%). EVI e fAPAR, por outro lado, apresentaram desempenhos semelhantes, classificando corretamente $93 \%$ e $92 \%$ das amostras analisadas, respectivamente.

Tabela 2 - Matriz de classificação (\% de acerto) para os quatro índices entre os dias 113 e 241 do ano (separabilidade baseada na distância de Mahalanobis).

\begin{tabular}{|c|c|c|c|c|}
\hline Alvos & NDVI & EVI & fAPAR & LAI \\
\hline Agricultura & 80 & 80 & 60 & 70 \\
\hline Cerrado arbóreo & 100 & 90 & 90 & 80 \\
\hline Cerrado arbustivo & 100 & 90 & 100 & 80 \\
\hline Cerrado herbáceo & 100 & 100 & 100 & 90 \\
\hline Floresta & 100 & 100 & 100 & 100 \\
\hline Pastagem & 100 & 100 & 100 & 80 \\
\hline Média & 97 & 93 & 92 & 83 \\
\hline \multicolumn{5}{|l}{}
\end{tabular}

\section{CONSIDERAÇÕES FINAIS}

As regiões de savanas respondem por, aproximadamente, $30 \%$ da produção primária (NPP) do mundo (IPCC, 1990). Desta forma, medidas precisas e acuradas da dinâmica sazonal das coberturas vegetais em áreas de savanas são de fundamental importância para se entender o funcionamento destes ecossistemas e implementar práticas de desenvolvimento sustentável.

Em fato, vários estudos têm demonstrado a sensibilidade dos índices de vegetação, em particular aqueles produzidos a partir dos dados do sensor MODIS (i.e. EVI e NDVI), às variações sazonais das várias fitofisionomias do bioma Cerrado e formas de uso associadas (Ratana et al., 2005). Contudo, até o momento, nenhum estudo, em escala de semidetalhe e abrangência regional, foi realizado com vistas a se avaliar o potencial do índice de área foliar (LAl) e da fração de radiação fotossinteticamente ativa absorvida (fAPAR) em responder à sazonalidade do Cerrado.

Assim, e tendo por base as paisagens predominantes no Cerrado Goiano, neste estudo avaliou-se a resposta e desempenho dos produtos M0D13A2 (EVI e NDVI) e MOD15A2 (LAI e fAPAR) em relação à variação sazonal e discriminação destas paisagens.

Ainda que os quatro índices MODIS tenham mostrado desempenho satisfatório e coerente quanto ao comportamento sazonal das fitofisionomias consideradas neste estudo, vale destacar a maior sensibilidade do LAl sobre os demais índices, e do EVI em relação ao NDVI.

Por outro lado, e no que diz respeito a separabilidade destas fitofisionomias, o melhor resultado de classificação foi do NDVI, com $97 \%$ de acerto, enquanto o uso do LAl resultou em maior confusão entre as classes, e índice de acerto geral de $83 \%$.

Estes resultados, ainda que preliminares, indicam que os produtos MOD13A2 e M0D15A2 respondem de forma diferenciada e complementar às variações sazonais e diferenças estruturais e funcionais encontradas nas varias paisagens do bioma Cerrado. Assim, as quatro variáveis disponibilizadas por estes produtos (i.e. EVI, NDVI, LAl e fAPAR) devem ser consideradas em estudos ecológicos / biofísicos, bem como em esforços voltados ao monitoramento sistemático e operacional deste complexo e ameaçado bioma.

\section{AGRADECIMENTOS}

Esta pesquisa foi parcialmente apoiada com recursos do projeto PROBIO Cerrado (MMA/CNPq/BIRD/GEF), através de uma bolsa de Desenvolvimento Tecnológico Industrial (RHAE - CNPq) concedida ao primeiro autor. 0 segundo autor é bolsista de produtividade do $\mathrm{CNPq}(\mathrm{PQ}-\mathrm{II})$.

\section{REFERÊNCIAS}

BRASIL. MINISTÉRIO DO MEIO AMBIENTE. 2003. Primeiro relatório nacional para a convenção sobre diversidade biológica Brasil. Serviços do ponto focal brasileiro do mecanismo de informação da convenção sobre diversidade biológica. Disponível em: <http://www.mma.gov.br/port/sbt/chm/relato.htm>. Acesso em: 03 abr. 2003 .

BITENCOURT MD, MESQUITA JR. HN, MANTOVANI W, BATALHA MA \& PIVELLO VR. 1997. Identificação de fisionomias de Cerrado com imagem índice de vegetação. In: Contribuição ao Conhecimento Ecológico do Cerrado, 1, 1997, Brasília. Anais do Congresso Brasileiro de Ecologia Brasilia: Universidade de Brasilia, Dep. de Ecologia, p. 316-320.

COHEN WB, MAIERSPERGER TK, YANG Z, GOWER ST, TURNER DP, RITTS WD, BERTERRETCHE M \& RUNNING SW. 2003. Comparisons of land cover and LAl estimates derived from ETM+ and MODIS for four 


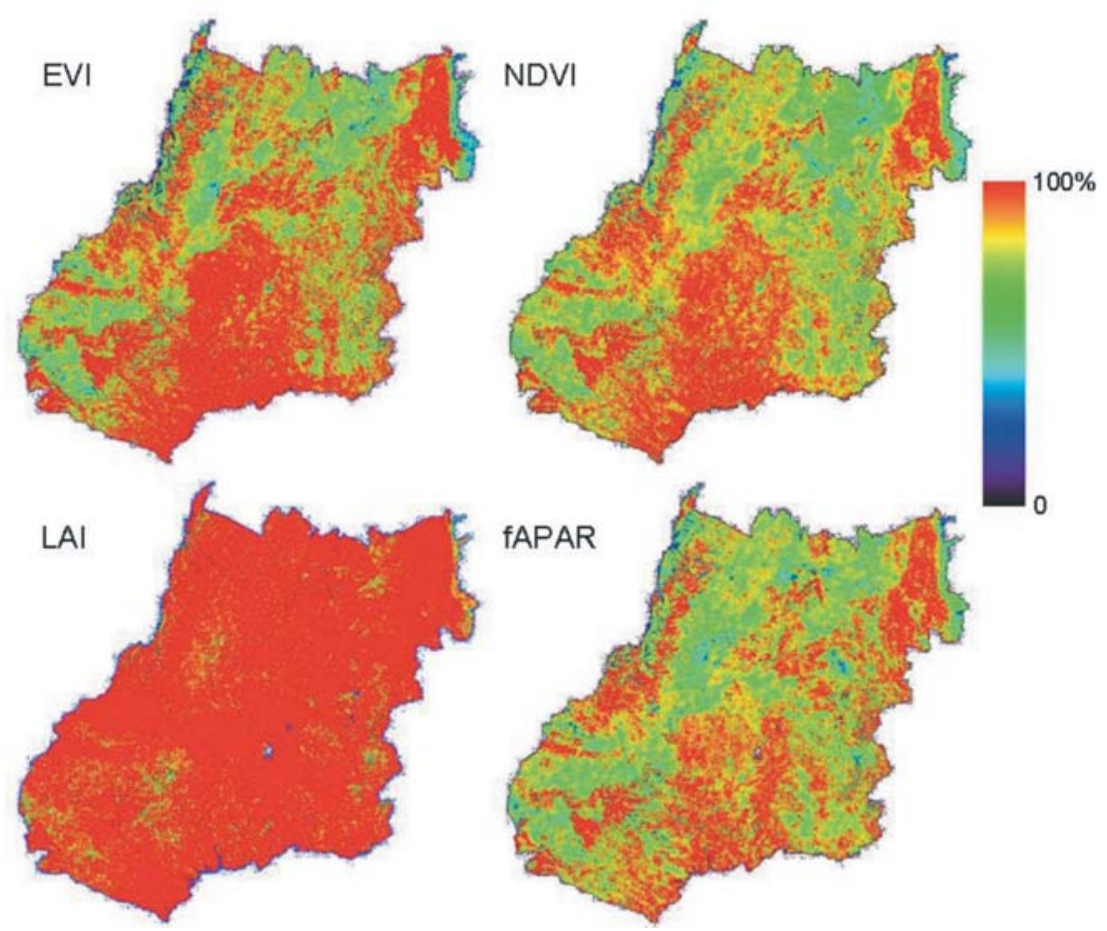

Figura 7 - Imagens da variação sazonal para os índices EVI, NDVI, fAPAR e LAl, obtidas a partir das respectivas diferenças relativas entre os dias do ano 113 e 241 (calendário Juliano).

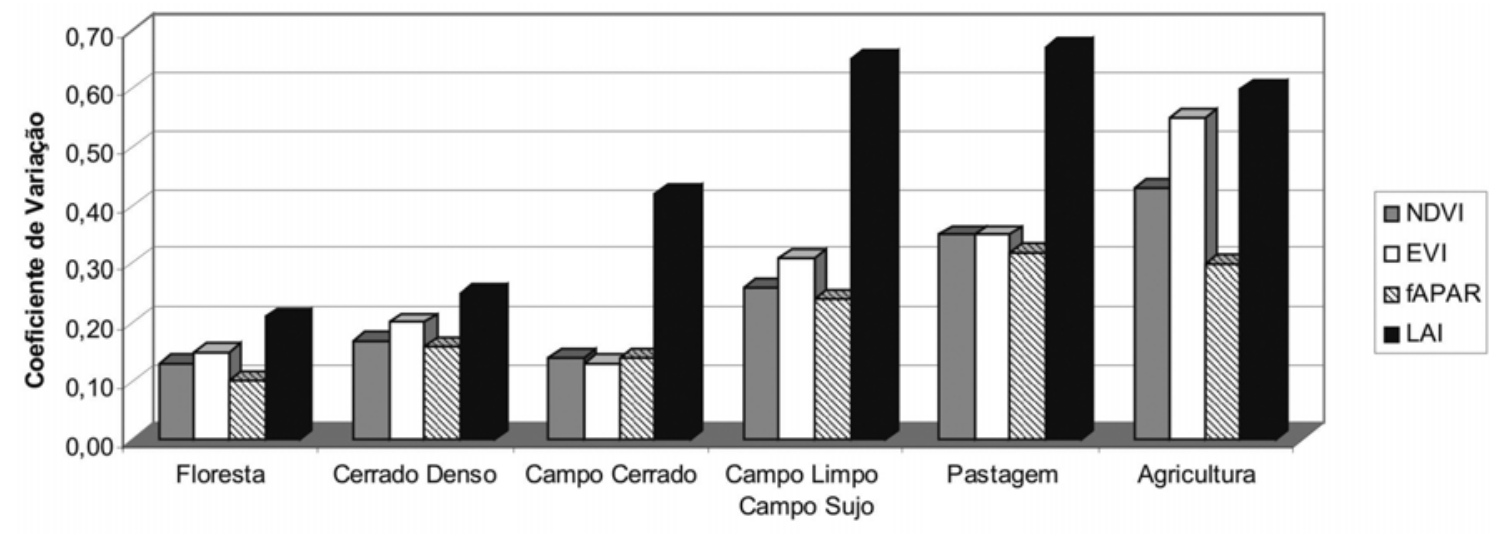

Figura 8 - Coeficientes de variação para os índices EVI, NDVI, LAI e fAPAR (dados referentes ao período entre dias do ano 113 e 241 - calendário Juliano) e respectivas classes de paisagens.

sites in North America: a quality assessment of 2000/2001 provisional MODIS products. Remote Sensing of Environment, 88: 233-255.

DIAS BFS. Cerrados: uma caracterização. 1992. In: DIAS BFS. (Org.). Alternativas de desenvolvimento dos Cerrados: manejo e conservação dos recursos naturais renováveis. Brasília: Fundação Pró-Natureza, p. 11-25.

DUARTE V, SHIMABUKURO YE \& AULICINO LCM. 2003. Metodologia para padronizar e atualizar o banco de dados do Projeto "PRODES Digital”. In: Simpósio Brasileiro de Sensoriamento Remoto, 11., 2003, Belo Horizonte, MG. Anais... São José dos Campos: INPE, 2003. p. 27052712. Repositório da URLib: <ltid.inpe.br/sbsr/2002/11.17.15.45>. Disponível em: <http://www.ltid.inpe.br/sbsr2003>. Acesso em: 10 nov. 2004.

EITEN G. 1972. The Cerrado vegetation of Brazil. The Botanical Review, New York, 38(2): 201-341. 
FENSHOLT R, SANDHOLT I \& RASMUSSEN MS. 2004. Evaluation of MODIS LAI, fAPAR and the relation between fAPAR and NDVI in a semiarid environment using in situ measurements. Remote Sensing of Environment, 91: 490-507.

FERREIRA LG, YOSHIOKA H, HUETE AR \& SANO EE. 2003. Seasonal landscape and spectral vegetation index dynamics in the Brazilian cerrado: An analysis within the Large-Scale Biosphere-Atmosphere Experiment in Amazônia. Remote Sensing of Environment, 87: 534-550.

FERREIRA NC, FERREIRA LG, HUETE AR \& FERREIRA ME. 2006. An operational deforestation mapping system using MODIS data and spatial context analysis. International Journal of Remote Sensing (in press).

HUETE AR, DIDAN K, MIURA T, RODRIGUEZ E, GAO X \& FERREIRA LG. 2002. Overview of the radiometric and biophysical performance of the MODIS vegetation indices (Special Issue). Remote Sensing of Environment, 83: 195-213.

IPCC. 1990. The IPCC scientific assessment of climate change. In: HOUGHTON JT, JENKINS GJ \& EPHARUMS JJ. (Eds.). Report of working group I. UK: Cambridge University Press, 365 pp.

JUSTICE CO, VERMONTE E, TOWNSHEND RD, ROY DP, HALL DK, SALOMONSON VV, PRIVETTE JL, RIGGS G, STRAHLER A, LUCHT W, MYNENI RB, KNYAZIKHIN Y, RUNNING SW, NEMANI RR, WAN Z, HUETE AR, VAN LEEUWEN WJD, WOLFE RE, GIGLIO L, MULLER J, LEWIS P \& BARNSLEY MJ. 1998. The moderate resolution imaging spectroradiometer (MODIS): Land remote sensing for global change research. IEEE Transactions on Geoscience and Remote Sensing, 36(4): 1-22.

LATRUBESSE EM, STEVAUX J \& SINHA R. 2005. Tropical rivers. Geomorphology, Netherlands, 70(3-4): 187-206.

MENDONÇA RC, FELFILI JM, WALTER BMT, SILVA MC, REZENDE AV, FILGUEIRAS TS \& NOGUEIRA PE. 1998. Flora vascular do Cerrado. In:
SANO SM \& ALMEIDA SP. (Ed.). Cerrado: ambiente e flora. Planaltina, DF: Embrapa Cerrados, p. 289-556.

MONTEIRO JMG. 1995. Fluxo de $\mathrm{CO}_{2}$ em um Cerrado sensu stricto. 1995. Dissertação (Mestrado) - Universidade de Brasília, Brasília. 61 f.

MYNENI RB, HOFFMAN S, KNYAZIKHIN Y, PRIVETTE JL, GLASSY J, TIAN Y, WANG Y, SONG X, ZHANG Y, SMITH GR \& LOTSCH A. 2002. Global products of vegetation leaf area and fraction absorbed PAR from year one of MODIS data. Remote Sensing of the Environment, 83(1-2): 214-231.

MYERS N, MITTERMEYER RA, MITTERMEIER CG, DE FONSECA GAB \& KENT J. 2000. Biodiversity hotspots for conservation priorities. Nature 403: 853-858.

PIVELLO VR \& COUTINHO LMA. 1996. Qualitative successional model to assist in the management of Brazilian cerrados. Forest Ecology and Management, Amsterdam, 87: 127-138.

RATANA P, HUETE AR \& FERREIRA LG. 2005. Analysis of Cerrado physiognomies and conversion in the MODIS seasonal - temporal domain. Earth Interactions (LBA Special Issue), 9: 1-22.

SANO EE, BARCELLOS AO \& BEZERRA HS. 2002. Assessing the spatial distribution of cultivated pastures in the Brazilian savanna. Pasturas Tropicales, Cali, 22(3): 2-15.

SCHOWENGERDT RA. 1997. Remote Sensing: models and methods for image processing. San Diego: Academic Press, $2^{\text {nd }}$ ed., 522 p.

WANG Y, WOODEOCK CE, BUERMANN W, STENBERG P, VOIPIO P, SMOLANDER H, HÄME T, TIAN Y, HU J, KNYAZIKCHIN Y \& MYNENI RB. 2004. Evaluation of the MODIS LAI algorithm at a coniferous forest site in Finland. Remote Sensing of Environment. 91: 114-127.

\section{NOTAS SOBRE OS AUTORES}

Manuel Eduardo Ferreira é Geógrafo, Mestre em Processamento de Dados em Geologia e Análise Ambiental (UnB) e Doutorando em Ciências Ambientais (CIAMB/UFG). É pesquisador-colaborador do Laboratório de Processamento de Imagens e Geoprocessamento (LAPIG/UFG), em projetos envolvendo o monitoramento de paisagens naturais e antrópicas nos biomas Cerrado e Amazônia, além de transferência de tecnologia. Seus interesses de pesquisa envolvem o aperfeiçoamento das técnicas de mapeamento e monitoramento da cobertura vegetal e uso do solo por meio do sensoriamento remoto de múltiplas resoluções.

Laerte Guimarães Ferreira Jr. é Geólogo, Mestre em Geologia Econômica e Doutor em Sensoriamento Remoto (Universidade do Arizona, EUA). Atualmente é pesquisador do CNPq (PQ II) e professor da Universidade Federal de Goiás junto ao Instituto de Estudos Sócio-Ambientais (IESA) e do Programa de Doutorado em Ciências Ambientais (CIAMB), além de coordenador do Laboratório de Processamento de Imagens e Geoprocessamento (LAPIG/UFG). Seus interesses de pesquisa envolvem o comportamento espectral de alvos naturais, além do mapeamento/monitoramento da paisagem (cobertura de solos e parâmetros biofísicos) com sensores ópticos de múltiplas resolucõoses.

Alfredo R. Huete é Professor titular do Departamento de Solo, Recursos Hídricos e Ciências Ambientais da Universidade do Arizona (EUA). Chefe do Laboratório de Biofísica Terrestre e Sensoriamento Remoto (TBRS). Principal Investigator do NASA MODLAND group (programa de aplicações terrestre do sensor MODIS) e do Projeto LBA (Large Scale Biosphere-Atmosphere Experiment in Amazon) e responsável pelo desenvolvimento dos índices de vegetação MODIS.

Alejandro Alvarado Peccinini é Biólogo, Mestre em Ecologia (USP). Atualmente é Gestor de Recursos Naturais na Agência Goiana do Meio Ambiente (AGMA). É colaborador de projetos envolvendo o monitoramento de Unidades de Conservação. Seus interesses de pesquisa envolvem os estudos sobre ecossistemas e Ecologia da Paisagem para o bioma Cerrado. 